\title{
Mejoras al ensayo de resistencia del enlace adhesivo de ligante asfáltico en materiales para pavimentos
}

\section{Improving reliability in the bitumen bond strength test on pavement materials}

\author{
Daybis Tencio González \\ Escuela de Ingeniería Química \\ Universidad de Costa Rica \\ San José, Costa Rica \\ daybis.tencio@ucr.ac.cr
}

\section{Alejandra Baldi}

Laboratorio Nacional de Materiales y Modelos Estructurales Universidad de Costa Rica

San José, Costa Rica

alejandra.baldi@ucr.ac.cr

\author{
José Pablo Aguiar Moya \\ Escuela de Ingeniería Civil \\ Universidad de Costa Rica \\ San José, Costa Rica \\ jose.aguiar@ucr.ac.cr
}

\author{
Bárbara Miranda Morales \\ Escuela de Ingeniería Química \\ Universidad de Costa Rica \\ San José, Costa Rica \\ barbara.mirandamorales@ucr.ac.cr
}

Fecha de recepción: 27 de mayo de 2021 / Fecha de aprobación: 07 de julio de 2021

\section{RESUMEN}

El estudio de las propiedades mecánicas de los materiales usados en pavimentos forma parte esencial de su caracterización inicial. Por ello, la reproducibilidad y la constante mejora de ensayos permite obtener resultados confiables, reduciendo errores que se puedan dar durante la preparación del material. Es por esto que el presente estudio permite establecer criterios y valores nominales para un adecuado acabado del agregado usado en pavimentos, con el fin de reducir la desviación de los resultados del ensayo de tracción uniaxial en base seca, realizados con ligante asfáltico virgen. Para el ensayo, se expuso a las muestras de agregado a un proceso de pulido por 60 segundos con un número de 10 pasadas, para lo cual se utilizaron bandas abrasivas de distintos números de grano, hasta el \#320. Se pudo obtener un esfuerzo de tracción de 2,39 $(0,29)$ $\mathrm{MPa}$, donde el valor entre paréntesis corresponde a la desviación estándar y el mismo corresponde a las condiciones donde se obtuvo un mejor esfuerzo de tracción, aunado a una disminución de desviación estándar. Adicionalmente, un modelo lineal entre los factores de preparación de superficie y el valor de resistencia de enlace adhesivo (POTS por sus siglas en inglés) obtenido por un diseño factorial $2^{4}$, mostró que los efectos principales aportan hasta un $84 \%$ de correlación con el esfuerzo de tracción y hasta un 78 \% con su desviación estándar, lo cual es considerado satisfactorio, ya que su valor p, el cual es una medida directa de la probabilidad de aceptar las condiciones recomendadas en este documento, es de $6,56 \cdot 10^{-4}$, demostrando una adecuada significancia estadística.

\section{ABSTRACT}

The study of mechanical properties of pavement materials is an essential part for their initial characterization. Therefore, the reproducibility and constant improvement of the tests involved allow to obtain faithful results, reducing the potential errors and variability that might occur during sample preparation. For this reason, this study focuses in establishing criteria and nominal values to obtain an adequate finish in the aggregate sources. This intends to reduce the standard deviation of the uniaxial tensile test, carried out by means of the bitumen bond strength test on dry conditions. The aggregates were treated by a sanding process for 60 seconds, repeating such cycles 10 times per sample. Also, abrasive bands of varying grain numbers were used. A Pull-Off Tensile Strength (POTS) of 2,39 (0,29) MPa was achieved, where the value in parentheses corresponds to the standard deviation. This value was selected due to the best conditions found in the experiment. Furthermore, a linear model obtained by a factorial design demonstrated the main effects provide up to $84 \%$ correlation with the pneumatic stress and up to $78 \%$ with its standard deviation, which is considered satisfactorily since the p-value defined in this research as the approval probability for the recommended conditions is $6,56 \cdot 10^{-4}$, demonstrating statistical significance.

Keywords: Bitumen bond strength test, pull off tensile stress, aggregate, sanding

Palabras clave: Ensayo de resistencia del enlace adhesivo, esfuerzo de tracción, agregado, pulido. 


\section{INTRODUCCIÓN}

Los fenómenos de cohesión y adhesión interfacial se han estudiado por diferentes autores, en especial, para materiales utilizados en la construcción de pavimentos, donde los fenómenos antes mencionados se pueden estudiar mediante dos enfoques distintos: el químico y el mecánico. Este estudio gira en torno del análisis mecánico de la adhesión en materiales como el agregado y el ligante asfáltico, donde se ha demostrado que la adhesión tiene un efecto importante en el desempeño y vida útil de los materiales.

Las propiedades adhesivas se pueden determinar directamente con técnicas para la medición del ángulo de contacto, como la energía superficial o el trabajo de desplazamiento del agua en interfaz ligante-agregado, o también otros métodos con acercamientos prácticos como el ensayo de resistencia del enlace adhesivo de ligantes asfálticos, BBS (del inglés Bitumen Bond Strength).

El ensayo BBS resulta ser un método ampliamente utilizado para cuantificar el enlace entre el ligante asfáltico y el sustrato sólido: el agregado. El BBS es una prueba selectiva ya que determina la adhesión mecánica de los materiales ensayados según sus propiedades mecánicas y reológicas, y según la sensibilidad que posea el material a la tracción uniaxial y a la humedad. El BBS tiene la capacidad de diferenciar entre la susceptibilidad a la humedad en diferentes muestras de ligantes asfálticos con diversas propiedades físicas y químicas (Sarkar, Rahman, Elseifi, Mayeux, y Cooper, 2020).

En la actualidad, esta es una prueba que se ha modificado para ser aplicada a ligantes asfálticos y se incorporó a la norma AASHTO T361 (Cardone, Virgili, y Graziani, 2018). Según Tausif y colaboradores (Tausif, Zaidi, Ahmad, y Jameel, 2020), el ensayo BBS se realiza bajo la modificación de las especificaciones de la norma ASTM D-4541.

En el ensayo BBS los punzones de extracción experimentan carga neumática en tracción y si esta carga es mayor a la energía de adhesión o cohesión mecánica, entonces, ocurre la falla del material en la interfase entre ligante y agregado (Tausif et al., 2020). La caracterización de dicha falla debe ser estudiada cuidadosamente, ya que depende directamente de un control visual estricto del espécimen fallado, la misma puede presentarse como falla cohesiva, falla adhesiva o una mezcla de ambas.

El ensayo BBS se evalúa tomando en cuenta tres factores principales: la fuerza de tracción (o POTS, del inglés Pull Off
Tensile Strength), el porcentaje de pérdida de la fuerza de tracción, y el modo de falla. La fuerza de tracción o POTS se puede calcular empleando la ecuación 1 (Das y Singh, 2018).

$$
\text { POTS }=\frac{\left(B P \cdot A_{g}\right)-C}{A_{p s}}
$$

donde,

POTS: fuerza de tracción, $\mathrm{kPa}$.

$B P$ : esfuerzo de rotura, $\mathrm{kPa}$.

$A_{g}$ : área de contacto entre la empaquetadura y la placa de reacción, $\mathrm{mm}^{2}$.

C: constante del equipo, $\mathrm{N}$.

$A_{p s}:$ área del punzón extraíble, $\mathrm{mm}^{2}$.

El efecto de la superficie de la muestra en el ensayo BBS es importante debido a que ésta afecta directamente las mediciones macroscópicas de la resistencia axial por la presencia de vacíos, imperfecciones o cualquier otro disturbio en la superficie.

En el ensayo se utilizan estos materiales usuales en pavimentos, donde se deposita una muestra de ligante asfáltico como agente adhesivo sobre el agregado mineral. La carga neumática uniaxial es aplicada sobre el punzón y el ensayo genera tres resultados principales:

1. El perfil de la fuerza de tensión a través del tiempo.

2. La resistencia máxima ante falla.

3. El tipo de falla por análisis visual o de imágenes.

Como se mencionó previamente, el ensayo BBS posee la particularidad de que la distribución de la carga neumática en las muestras permite tres posibles modos de falla: adhesión, cohesión o una combinación de ambos. El modo de falla es el resultado de la resistencia interfacial máxima permitida por los materiales durante el esfuerzo proporcionado por la carga.

Con respecto a la adhesión entre dos superficies, ésta se define como el proceso en donde partículas o superficies dispares se encuentran unidas por fuerzas de valencia o fuerzas de enclavamiento (Moraes, Velasquez, y Bahia, 2011). Por tanto, una mala adhesión interfacial en una mezcla asfáltica en presencia de agua afectará sus propiedades, teniendo algunas consecuencias mecánicas como: 
1. Pérdida de cohesión en la mezcla asfáltica.

2. Falla del enlace adhesivo entre el agregado y el ligante asfáltico, también se conoce como desnudamiento.

3. Degradación del agregado.

En consecuencia, el daño por humedad contribuye altamente al agrietamiento del pavimento, mediante cinco mecanismos que son: desprendimiento, desplazamiento, emulsificación espontánea, presión de poros e hidráulica (Strategic Highway Research Program, 1991).

A este punto se resalta la necesidad de disminuir la variabilidad del ensayo de tracción unixial al ser un indicador directo del esfuerzo de tracción interfacial entre agregado y ligante asfáltico. Por tanto, el objetivo del presente estudio es determinar las condiciones de preparación superficial que mejoren los resultados del ensayo BBS. Este objetivo se puede lograr minimizando inicialmente la desviación estándar y obteniendo las mejores condiciones para ese propósito. Posteriormente, se deben registrar las condiciones que maximicen el esfuerzo de enlace del ligante y contrastar ambas condiciones para determinar la preparación superficial adecuada para el agregado mineral.

\section{Aplicabilidad}

Es importante detallar que el ensayo BBS puede determinar el esfuerzo de tracción en condiciones de humectación completa, por lo que se puede determinar el porcentaje de pérdida de fuerza del enlace de adhesión, el cual es un parámetro de estudio que pretende estimar la resistencia al daño por humedad del material y se calcula mediante la ecuación 2 (Das y Singh, 2018):

$\%$ de pérdida de fuerza del enlace $=\frac{\text { POTS }_{\text {seco }}-\text { POTS }_{\text {húmedo }}}{P O T S_{\text {seco }}}$

El esfuerzo de tracción en seco se mide a temperatura ambiente, mientras que para la fuerza de tracción en húmedo se necesita agua a $40{ }^{\circ} \mathrm{C}$. En el caso del último parámetro, el modo de falla se determina con inspección visual de la superficie del agregado (Das y Singh, 2018).

Tanto la determinación en base seca como húmeda dependen de la preparación de la superficie de la muestra de agregado, debido al esparcimiento del ligante sobre la misma, donde los vacíos internos e imperfecciones superficiales pueden llegar a exponer nuevas microestructuras de soporte después de un pulido agresivo y descontrolado. Estas microestructuras afectan el valor de esfuerzo durante la ejecución del ensayo tal como se observa en la Figura 1. Razón por la cual es importante estudiar las condiciones de preparación de superficie y su efecto sobre la resistencia de enlace adhesivo de estos materiales.

a)

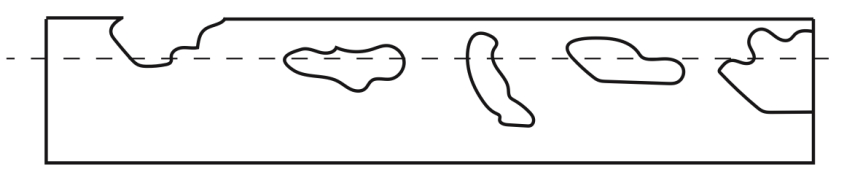

b)

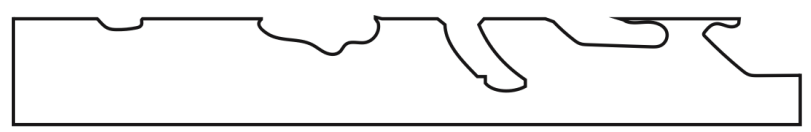

Figura 1. Vacíos internos expuestos en la superficie una vez que se pule, a) muestra de agregado mineral sin pulir y b) muestra de agregado mineral pulida

Con lo anterior, se observa que la preparación de la superficie es un factor importante para el ensayo BBS por lo que controlar, estudiar y buscar las mejores condiciones para las variables de pulimiento como: ciclos de pulido, grano nominal y tiempo de exposición provocarían una reducción de la variabilidad del ensayo y un mejor desempeño de esta.

\section{METODOLOGÍA}

Tanto los requerimientos como equipos necesarios para el ensayo de tracción uniaxial se describen en la norma ASTM D4541-17, o bien, su correspondiente AASHTO T361. En resumen, se tomó una muestra de agregado con dimensiones de $140 \mathrm{~mm} \cdot 70 \mathrm{~mm} \cdot 10 \mathrm{~mm}$ para un espaciamiento entre punzones de $35 \mathrm{~mm}$, luego se agregó 0,40 g de ligante asfáltico y se procedió a armar el equipo para su posterior falla, como se muestra en la Figura 2. Para un mayor detalle sobre el protocolo puede encontrarse más información en el proyecto de graduación titulado "Determinación de las mejores condiciones de preparado de muestra para las pruebas de energía superficial y adhesión neumática en agregado con análisis topológico superficial para la generación de un protocolo" (Tencio, 2019). 
a)

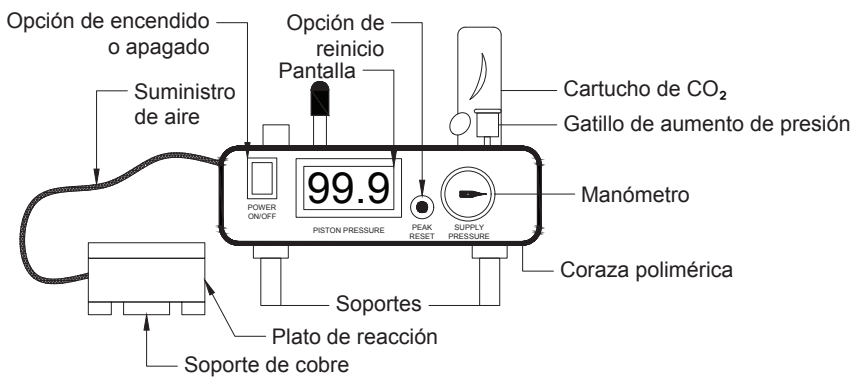

b)

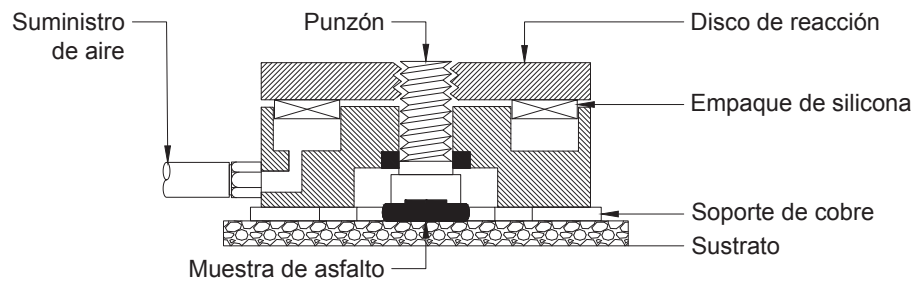

Figura 2. Descripciones gráficas de las partes que conforman el ensayo enlace adhesivo con agregado y ligante asfáltico

Las condiciones superficiales se regulan según un set de variables en la preparación del pulido; las cuales son: tipo de agregado, grano nominal, corridas y tiempo de exposición. En el Cuadro 1 se detalla cada una de las variables del diseño factorial $2^{4}$.

Las condiciones mencionadas en el Cuadro 1 fueron escogidas luego de efectuar estudios de sensibilidad del ensayo de BBS. Lo anterior fue necesario ya que tal ensayo tiene un carácter destructivo para las muestras, por lo que se consideró variar los niveles de los factores a analizar y elegir aquellos que tuviesen un efecto en el valor de POTS.

Con el fin de obtener las mejores condiciones que minimicen la variabilidad del ensayo, así como conocer los efectos principales e interacciones con las variables respuesta del ensayo, se utiliza un diseño de experimentos tipo factorial con 2 niveles y 4 factores.

El pulido superficial se debe realizar de manera progresiva; es decir, se debe pulir la superficie con cada banda abrasiva existente hasta alcanzar el valor nominal señalado en el diseño estadístico del Cuadro 1 . Una vez que se obtienen las distintas condiciones superficiales para el agregado mineral, este se limpió y acondicionó.
La limpieza inicia al colocar las muestras de agregado con agua destilada dentro de un baño ultrasónico durante 60 minutos a $60{ }^{\circ} \mathrm{C}$, con el fin de evitar que impurezas y agregados finos se encuentren en la superficie. Una vez que la muestra se encuentre limpia, se procede a secar la muestra en un horno de convección a $60^{\circ} \mathrm{C}$ durante 3 horas.

En lo que respecta a la preparación de la interfase liganteagregado, se utilizó un solo tipo de ligante asfáltico virgen y se colocaron $0,40 \mathrm{~g}$ de ligante asfáltico en la cabeza de cada punzón y se esperó a que se dispersara adecuadamente. Posteriormente, los punzones se colocaron sobre la muestra de agregado manteniendo una distancia entre ellos de $35 \mathrm{~mm}$.

Luego, las muestras se dejaron reposar hasta que alcancen la temperatura ambiente y se calibra el equipo marca Quantum Gold para el ensayo BBS, en el cual se procede a dar paso de una corriente neumática que genera tracción sobre el punzón para alcanzar una tasa de aumento de presión constante. Luego de ello, se procede a realizar el ensayo a cada muestra con ligante asfáltico monitoreando y almacenando la presión durante el ensayo hasta el fallo de éste. Es importante mencionar que el ligante asfáltico se preparó según la norma AASHTO T361 (equivalente a ASTM D4541), no se omite indicar que a la fecha de este estudio no existe una correspondencia nacional para dicho ensayo.

Cuadro 1. Descripción de las variables de pulimiento del agregado para el diseño factorial $2^{4}$

\begin{tabular}{|c|c|c|c|c|}
\hline Factor & Símbolo & Descripción & Nivel bajo & Nivel alto \\
\hline Tipo de agregado & $\mathrm{M}$ & Naturaleza química del agregado mineral & Silícico \\
\hline Grano nominal & $\mathrm{N}$ & $\begin{array}{c}\text { Tamaño de grano nominal de la banda abrasiva } \\
\text { (banda abrasiva) }\end{array}$ & $\# 120$ \\
\hline Corridas & $\mathrm{R}$ & $\begin{array}{c}\text { Número de veces que la banda abrasiva se pasa } \\
\text { sobre la superficie de la muestra }\end{array}$ & 1 vez & 10 veces \\
\hline Tiempo de exposición & $\mathrm{t}$ & Duración de cada corrida & 10 segundos & 60 segundos \\
\hline
\end{tabular}


Las determinaciones de rugosidad constan del uso de un perfilómetro de contacto marca Bruker modelo Dektak XT el cual utiliza una punta de cuarzo donde la misma mapea una superficie cuadrada de $2500 \mu \mathrm{m}$ con una resolución de $0,833 \mu \mathrm{m} / \mathrm{pt}$ durante $10 \mathrm{~s}$ por traza y un total de 500 trazas, se utilizó una fuerza de $2 \mathrm{mg}_{\mathrm{f}}$ basada en criterios del fabricante $\mathrm{y}$ desgaste de la punta de cuarzo sobre la muestra de agregado.

En el Cuadro 2 se muestra la identificación de las muestras con los niveles utilizados en el experimento para cada una de las variables en estudio.

\section{RESULTADOS Y DISCUSIÓN}

La norma AASHTO T361 que establece el protocolo del ensayo $\mathrm{BBS}$ indica que la preparación de la superficie del agregado se debe realizar con una banda abrasiva cuyo tamaño de grano nominal es 280. Por esta razón, en este estudio se eligieron valores de banda abrasiva de 120 y 320 como niveles bajo y alto, respectivamente, de modo que se obtuviese un acabado superficial más pulido y otro más rugoso que el indicado en la norma. Lo anterior permite analizar si existe un efecto en los valores de POTS y en la desviación estándar resultante al ensayar muestras de agregado con distintos acabados.
Con el objetivo de dimensionar la calidad del acabado superficial, se midió la rugosidad de cada muestra, permitiendo así conocer si en efecto los tratamientos aplicados generan superficies diferentes en el agregado. En el Cuadro 3 se muestran los resultados del ensayo BBS y se incluye cada valor obtenido de rugosidad absoluta. Es importante resaltar que los tipos de agregados utilizados en este estudio corresponden a las dos fuentes principales existentes en el país, silícica y caliza, siendo esta última poco utilizada en mezclas asfálticas. Adicionalmente, fueron seleccionadas ya que difieren en la resistencia al pulimiento de cada una, ya que la caliza es altamente conocida por pulirse con facilidad.

De acuerdo con los resultados del Cuadro 3, se observa que algunas muestras con menos rugosidad suelen mostrar valores elevados de POTS. Este fenómeno es ocasionado por la reducción de material en la superficie tras el pulido, lo que genera la exposición de vacíos internos que aparecen en la superficie de la muestra y pueden dar paso a la aparición de nuevas imperfecciones; como se observa en la Figura 1. Esto significa que cada vez que se exponen más defectos los mismos ejercen nuevos micro-soportes ante la acción del esfuerzo neumático en tracción. Cabe destacar que este efecto no fue cuantificado debido a que depende enteramente de la topología de los vacíos y de los defectos expuestos en el material.

Cuadro 2. Descripción de las muestras en el diseño experimental utilizado

\begin{tabular}{|c|c|c|c|c|}
\hline Iden & Grado nominal, $N$ & Tiempo de exposición, $t /(s)$ & Tipo de agregado, M & Cantidad de carreras, C \\
\hline A1 & 320 & 10 & Calizo & 1 \\
\hline A2 & 320 & 10 & Calizo & 1 \\
\hline A3 & 320 & 10 & Silícico & 10 \\
\hline A4 & 320 & 10 & Calícico & 1 \\
\hline A5 & 320 & 60 & Calizo & 10 \\
\hline A6 & 320 & 60 & Silícico & 1 \\
\hline A7 & 320 & 60 & Silícico & 10 \\
\hline A8 & 320 & 60 & Calizo & 1 \\
\hline B1 & 120 & 10 & Calizo & 1 \\
\hline B2 & 120 & 10 & Silícico & 1 \\
\hline B3 & 120 & 10 & Silícico & 1 \\
\hline B4 & 120 & 10 & Calizo & 1 \\
\hline B5 & 120 & 60 & Calizo & 10 \\
\hline B6 & 120 & 60 & Silícico & 1 \\
\hline B7 & 120 & 60 & Silícico & 10 \\
\hline B8 & 120 & 60 & & 10 \\
\hline
\end{tabular}


Cuadro 3. Valores de fuerza de tracción (POTS), desviación estándar y rugosidad absoluta asociadas a las interfaces de agregado y ligante asfáltico

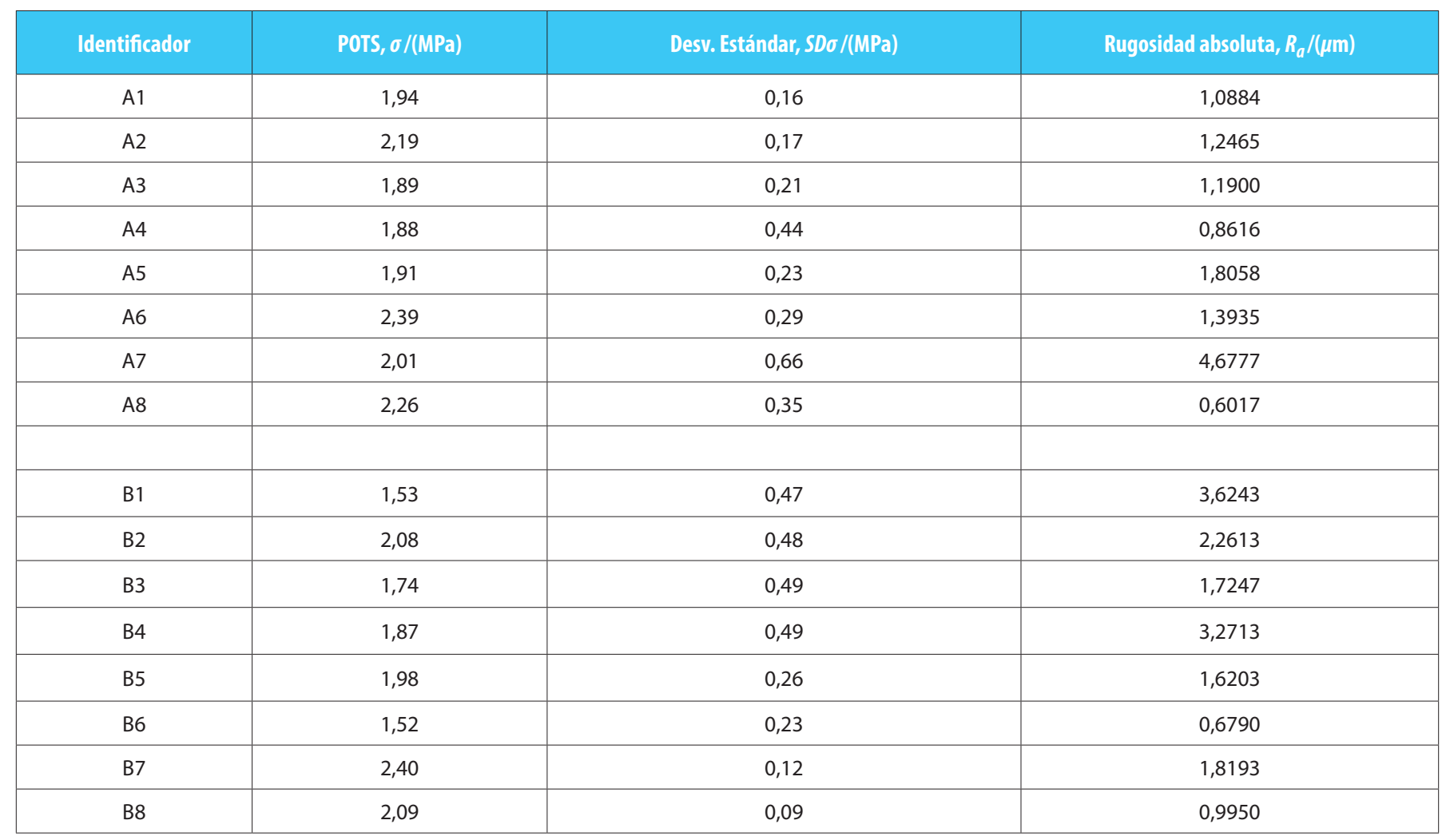

Otro factor que afecta el caso de estudio es la heterogeneidad composicional de los agregados, la cual afecta la tracción mecánica debido a la diferencia de dureza y propiedades físicas de éstos y los misceláneos dentro del agregado, ya que la muestra al contener diferentes compuestos, estos ejercen una interacción adhesiva diferenciada, para la cual se recomienda mapear con el fin de determinar su efecto. A pesar de estas diferencias, los valores de rugosidad y POTS demuestran que, para la mayoría de las muestras, el nivel alto de \#320 de grano nominal de banda abrasiva usada en la preparación superficial produce una superficie con mejor adhesión.

Ahora bien, en la Figura 3 se observa que la variación en la cantidad de corridas (entre 1 y 10) produce un aumento promedio en POTS de 0,138 MPa; si se cambia el tiempo de exposición de cada corrida de $10 \mathrm{~s}$ a $60 \mathrm{~s}$, el aumento es de 0,188 MPa. Por su parte, el número de grano nominal aumenta la fuerza de tracción en 0,163 $\mathrm{MPa}$, cuando se cambian las bandas de abrasión de \#120 a \#320. De acuerdo con el análisis de sensibilidad anterior, se puede observar que el efecto del tiempo de exposición tiene gran impacto sobre el valor del esfuerzo de adhesión, esto indica que si se toma mucho más tiempo para preparar la superficie se necesitará mayor energía para la ruptura lo cual es beneficioso en la mezcla asfáltica. La Figura 3b muestra que si se usan menor cantidad de ciclos de pulimiento la desviación estándar disminuirá importantemente, dato que se puede observar en el valor $\mathrm{p}$ en el Cuadro 4 y el Cuadro 5.

Con el objetivo de analizar el efecto que tiene cada uno de los factores sobre el valor de la fuerza de tracción para cada combinación, se propone un modelo estadístico, el cual se detalla en el Cuadro 4. El modelo descrito busca relacionar los valores de POTS obtenidos, y la desviación estándar asociada, con los factores analizados.

En el Cuadro 4 se aprecia que, de acuerdo con el diseño factorial aplicado, el factor tiempo de exposición tiene el mayor efecto sobre el valor de POTS, ya que posee el valor p más bajo, lo que indica que existe confianza suficiente para afirmar que tiene una mayor significancia estadística. 


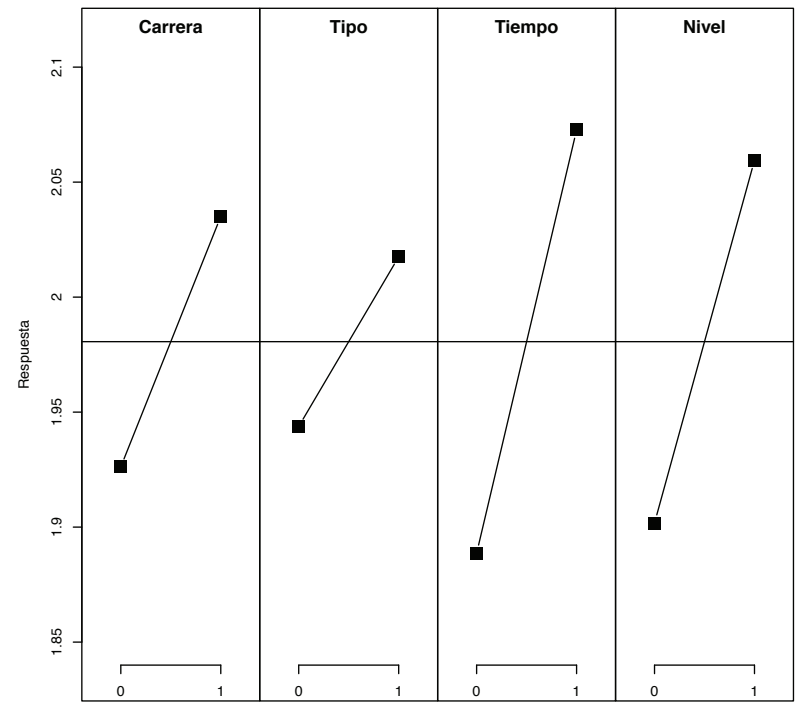

b)

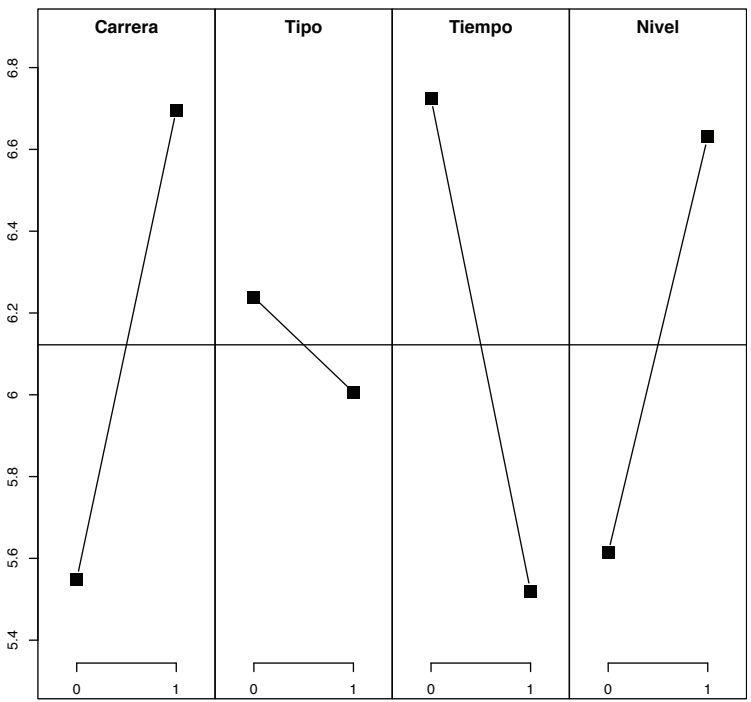

Figura 3. Análisis de sensibilidad y comportamiento de los efectos para: a) Resistencia de enlace adhesivo, b) Desviación estándar

Cuadro 4. Modelo estadístico con coeficientes $w_{i}$ lineales para la medición de la resistencia de enlace adhesivo ( $\sigma$ ) y su desviación estándar (SD $\left.\sigma\right)$

\begin{tabular}{|c|c|c|c|c|c|c|}
\hline \multirow{3}{*}{ Modelo Lineal } & \multicolumn{3}{|c|}{$\frac{\sigma}{(M P a)}=\sum \omega_{i} E_{i}+\epsilon$} & \multicolumn{3}{|c|}{$\frac{S D_{\sigma}}{(M P a)}=\sum \omega_{i}^{*} E_{i}+\epsilon$} \\
\hline & \multicolumn{3}{|c|}{$\begin{array}{l}\text { Residuo, } \varepsilon \\
0,9197\end{array}$} & \multicolumn{3}{|c|}{$\begin{array}{l}\text { Residuo, } \varepsilon \\
3,466\end{array}$} \\
\hline & $\begin{array}{l}p \text {-value } \\
9,83 \times 10^{-5}\end{array}$ & $\begin{array}{l}F_{4,12 d f} \\
15,85\end{array}$ & $\begin{array}{c}R^{2} \\
0,8408\end{array}$ & $\begin{array}{l}p \text {-value } \\
6,56 \times 10^{-4}\end{array}$ & $\begin{array}{l}F_{4,12 \text { df }} \\
10,59\end{array}$ & $\begin{array}{c}R^{2} \\
0,7793\end{array}$ \\
\hline Factor, $E_{i}$ & Estimado, $w_{i}\left(S D_{w}\right)$ & t-value & $\operatorname{Pr}(>|t|)$ & Estimado, $w_{i}^{*}\left(S D_{w^{*}}\right)$ & $\mathrm{t}$-value & $\operatorname{Pr}(>|t|)$ \\
\hline Corridas, $R$ (número de veces) & $0,7963(0,41)$ & 1,936 & 0,0768 & $3,451(1,550)$ & 2,226 & 0,0459 \\
\hline Tipo de agregado, $M$ (adimensional) & $0,7611(0,41)$ & 1,850 & 0,0890 & $2,071(1,550)$ & 1,336 & 0,2063 \\
\hline Tiempo de exposición, $T(\mathrm{~s})$ & $0,8714(0,41)$ & 2,119 & 0,0557 & $1,098(1,550)$ & 0,708 & 0,4923 \\
\hline Grano nominal, $N(\#)$ & $0,8451(0,41)$ & 2,055 & 0,0624 & $3,321(1,550)$ & 2,142 & 0,0534 \\
\hline
\end{tabular}

Por otro lado, los resultados muestran que una banda abrasiva de grano nominal \#320 posee un valor mayor de fuerza de tracción y que la fiabilidad del ensayo BBS es mayor que en una superficie que se encuentra preparada de forma menos detallada (niveles bajos del diseño factorial). Lo anterior se puede ver evidenciado en el Cuadro 3, donde los valores de carrera menores producen una disminución promedio considerablemente menor de la desviación estándar, alrededor de $0,32 \mathrm{MPa}$.

En general, el modelo propuesto para la fuerza de tracción posee una relación del $84 \%$ entre el valor medido experimentalmente y el estimado de acuerdo con el diseño factorial. Asimismo, el valor p que se determinó para el modelo permite establecer con una confianza estadística significativa que el modelo tiene validez. El mismo análisis se realizó para la desviación estándar y se determinó que las condiciones presentadas por el modelo satisfacen la disminución de la desviación estándar $y$, por ende, permiten una reproducibilidad fiel del ensayo.

Es importante indicar que no todos los efectos individuales del diseño experimental afectan de forma positiva, es decir incrementando el valor de POTS y disminuyendo la desviación estándar. Adicionalmente, en este estudio no se encontró interacción entre los efectos por lo que el modelo lineal se considera estadísticamente suficiente para estimar el valor de POTS y la desviación estándar para las interfases de agregado y ligante virgen. El análisis estadístico realizado permitió establecer las mejores condiciones de preparación de muestra de agregado para el ensayo BBS (Cuadro 5). 
Cuadro 5. Condiciones recomendadas de preparación superficial para ambos agregados calizo y silícico para el ensayo BBS

\begin{tabular}{|c|c|c|}
\hline \multirow{2}{*}{ Factor, $E_{i}$} & \multicolumn{2}{|c|}{ Niveles de los factores } \\
\cline { 2 - 3 } & POTS & Desviación Estándar \\
\hline $\begin{array}{c}\text { Corridas, } \\
R \text { (número de veces) }\end{array}$ & 10 & 1 \\
\hline $\begin{array}{c}\text { Tiempo de exposición, } \\
t(\mathrm{~s})\end{array}$ & 60 & 10 \\
\hline \begin{tabular}{c} 
Grano nominal, $N(\#)$ \\
\hline
\end{tabular} & 320 & 320 \\
\hline
\end{tabular}

En el Cuadro 5, se aprecian los resultados del diseño factorial, los cuales revelan que las mejores condiciones para el valor de POTS desde el punto de vista estadístico para ambos agregados minerales son: 10 corridas por cada banda abrasiva usada, durante 60 segundos de tiempo de exposición y con un acabado final logrado con el abrasivo \#320.

Mediante el análisis del diseño experimental se encontró que no existe diferencia en las condiciones de preparación de la superficie para ambos tipos de agregado mineral para la medición de POTS. Lo cual implica que ambos tipos de agregado, calizo o silícico, pueden prepararse de la misma manera sin variar los resultados de POTS que se obtienen.

Finalmente, en la Figura 4 se puede observar que las condiciones de preparación que permiten obtener valores de POTS con menor desviación estándar son: 1 corrida de duración de 10 segundos y un acabado final dado con la banda \#320.

Es importante resaltar que las condiciones de nivel bajo representan un tratamiento descuidado de la superficie, en donde el pulimiento se hace de manera rápida, mientras que el nivel alto representa un tratamiento más detallado, buscando una superficie más homogénea. En consecuencia, y de acuerdo con el Cuadro 5, los valores de POTS se ven mejorados al aplicar un acabado superficial correspondiente a los niveles altos de corridas y tiempo de exposición, mientras que la desviación estándar del ensayo se reduce al aplicar un tratamiento con niveles bajos de estos mismos factores.

Se encontró que el mejor grano nominal de abrasión correspondió a la banda abrasiva de grano nominal \#320 en ambos casos y se determinó que el tipo de banda es importante en la preparación superficial mediante la comparación del valor $\mathrm{p}$ relacionado con dicho efecto $(\mathrm{p}=0,0534)$.

Por lo tanto, se logró disminuir la desviación estándar del ensayo BBS para diferentes acabados superficiales, esto se deduce al observar la gran disminución de la desviación estándar de los valores de POTS. Por lo que, el tratamiento descrito para las muestras de agregado bajo las condiciones analizadas y resumidas en el Cuadro 5, sirven de insumo para el establecimiento de un protocolo de preparación, adicional al estándar actual. a)

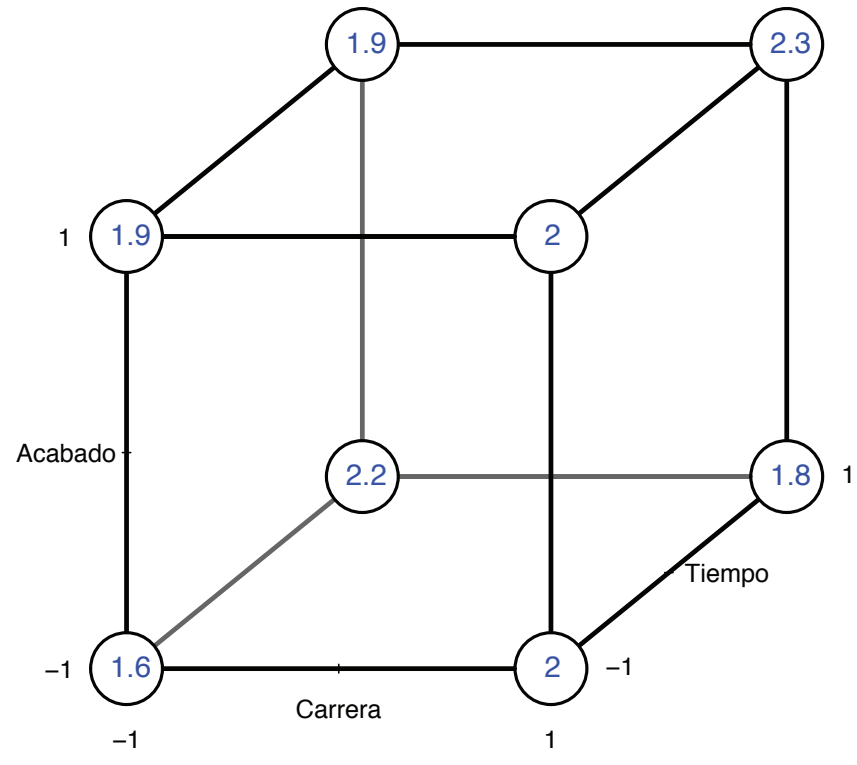

b)

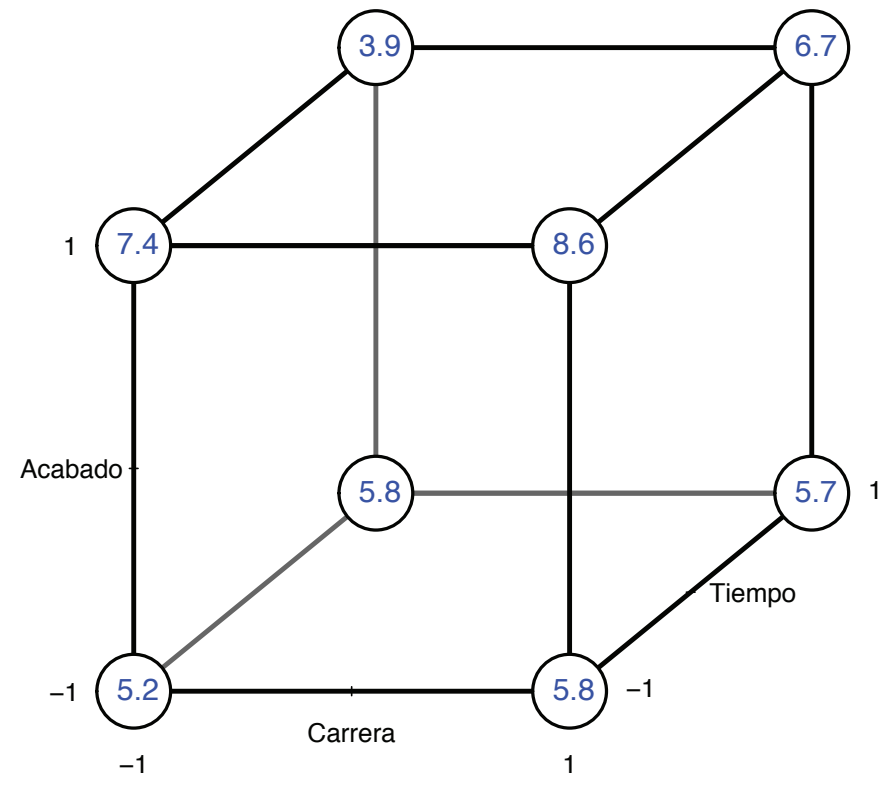

Figura 4. Gráfico de cubo del diseño factorial para: a) Resistencia de enlace adhesivo, b) Desviación estándar. 


\section{CONCLUSIONES}

Los hallazgos del presente estudio permiten concluir:

- Las condiciones recomendadas de preparado superficial para mejorar el valor de POTS de los agregados minerales estudiados son 10 corridas en cada banda abrasiva por debajo e incluyendo la banda \#320 con un tiempo de exposición a la banda de $60 \mathrm{~s}$ en cada corrida.
- La operación de preparado superficial combinado con las irregularidades superficiales preexistentes, vacíos superficiales, imperfecciones y porosidad determinan la calidad del acabado final de la muestra de agregado.

- A partir de una preparación adecuada de las muestras de agregado (sin diferenciar entre calizo o silícico) es posible mejorar la calidad de los datos de POTS en base seca obtenidos, lo cual se traduce en una reducción de la desviación estándar asociada a los mismos.

\section{REFERENCIAS}

The American Association of State Highway and Transportation Officials (2020). Standard Method of Test for Determining Asphalt Binder Bond Strength by Means of the Binder Bond Strength (BBS) Test (AASHTO T361-16).

ASTM International (2018). Standard Test Method for Coating Flexibility of Prepainted Sheet (D4145-10). DOI: 10.1520/D414510R18

Cardone, F., Virgili, A., y Graziani, A. (2018). Evaluation of bonding between reclaimed asphalt aggregate and bitumen emulsion composites. Construction and Building Materials, 184, 565-574. DOI: 10.1016/j.conbuildmat.2018.07.016

Das, A., y Singh, D. (2018). Effects of Regular and Nano Sized Hydrated Lime Fillers on Fatigue and Bond Strength Behavior of Asphalt Mastic. Transportation Research Record, 2672(28), 31-41. DOI: 10.1177/0361198118759064.

Moraes, R., Velasquez, R., y Bahia, H. U. (2011). Measuring the effect of moisture on asphalt-aggregate bond with the bitumen bond strength test. Transportation Research Record 2209(1), 70-81. DOI: 10.3141/2209-09

Sarkar, M., Rahman, M., Elseifi, M., Mayeux, C., y Cooper, S. (2020). Rheological and Molecular Characterizations of Tire Rubber Modified Asphalt Emulsion. Transportation Research Record, 2674(3), 12-26. DOI: 10.1177/0361198120908871

Strategic Highway Research Program (1991). The effect of the physical and chemical characteristics of the aggregate on bonding (SHRP-A/UIR-91-507). Recuperado de: http://onlinepubs.trb.org/onlinepubs/shrp/SHRP-91-507.pdf

Tausif, M., Zaidi, S., Ahmad, N., y Jameel, M. (2020). Influence of Natural Zeolite and Paraffin Wax on Adhesion Strength Between Bitumen and Aggregate. Civil Engineering Journal, 6(4), 733-742. DOI: 10.28991/cej-2020-03091505.

Tencio, S. (2019). Determinación de las mejores condiciones de preparado de muestra para las pruebas de energía superficial y adhesión neumática en agregado con análisis topológico superficial para la generación de un protocolo (Tesis de grado). Universidad de Costa Rica, San José. 